\title{
Thicker carotid intima-media thickness and increased plasma VEGF levels suffered by post-acute thrombotic stroke patients
}

This article was published in the following Dove Press journal:

International Journal of General Medicine

14 December 2016

Number of times this article has been viewed

\author{
Yuyun Yueniwati' \\ Ni Komang Darmiastini' \\ Eko Arisetijono ${ }^{2}$ \\ 'Radiology Department, Faculty of \\ Medicine, Brawijaya University, Malang, \\ Indonesia; ${ }^{2}$ Neurology Department, \\ Faculty of Medicine, Brawijaya \\ University, Malang, Indonesia
}

Background and objectives: Atherosclerosis causes reduction of the oxygen supply to structures in the far arterial wall, provoking the release of factors that drive angiogenesis of vasa vasorum, including VEGF. Other studies have revealed the inflammatory response in atherosclerosis and the role of platelet factor 4 (PF4) as an anti-angiogenic chemokine through the inhibition of VEGF. This cross-sectional study aims at measuring the effect of atherosclerosis assessed through carotid intima-media thickness (CIMT) against plasma VEGF levels in patients with post-acute thrombotic stroke.

Materials and methods: CIMT was assessed sonographically using GE Logiq S6 with 13 $\mathrm{MHz}$ frequency linear probe. VEGF-A plasma levels were measured using enzyme-linked immunosorbent assay (ELISA) method. Differences among variables were compared statistically. The data were analyzed using Pearson correlation.

Results: A total of 25 patients with post-acute thrombotic stroke were identified in days 7 to 90 . CIMT thickening was indicated in $88 \%$ of patients $(1.202 \pm 0.312 \mathrm{~mm})$, while an increase in plasma VEGF was identified in all patients $(178.28 \pm 93.96 \mathrm{ng} / \mathrm{mL})$. There was no significant correlation between CIMT and plasma VEGF levels in patients with post-acute thrombotic stroke ( $p=0.741)$. A significant correlation was recognized between CIMT and total cholesterol $(p=0.029)$ and low-density lipoprotein $(p=0.018)$.

Conclusion: There were no significant correlations between CIMT and plasma VEGF levels in patients with post-acute thrombotic stroke. However, plasma VEGF increased in patients with thrombotic stroke. CIMT measurement is a promising noninvasive modality to assess the vascular condition of patients with stroke and diabetes, while plasma VEGF cannot specifically assess vascular condition as it can be triggered by ischemic conditions in tissues of the whole body.

Keywords: atherosclerosis, CIMT, VEGF, thrombotic stroke

\section{Introduction}

Stroke is the leading cause of death and disability worldwide. In Indonesia, the average age of stroke patients is 58.8 years old and stroke is the leading cause of a high mortality rate in people above 50 years old. ${ }^{1-3}$ The number of stroke patients is inclined to increase every year, not only among elderly people, but also young and productive people. ${ }^{4}$ The most common type of stroke is thrombotic stroke with atherosclerosis as the most common cause. ${ }^{5,6}$ In atherosclerosis, thickening of the intima causes a decrease in the supply of oxygen to the far arterial wall, causing ischemia in blood vessels which provokes the release of factors stimulating angiogenesis of vasa vasorum, such as EPO, VEGF, and Ets-1. ${ }^{7}$ Other studies have shown the existence of inflammatory
Correspondence: Yuyun Yueniwati Radiology Department, Faculty of Medicine, Brawijaya University, Jl Veteran Malang, 65I45, Indonesia

Tel +62818381800

Fax +62 34I 34I 808

Email yuyun@ub.ac.id 


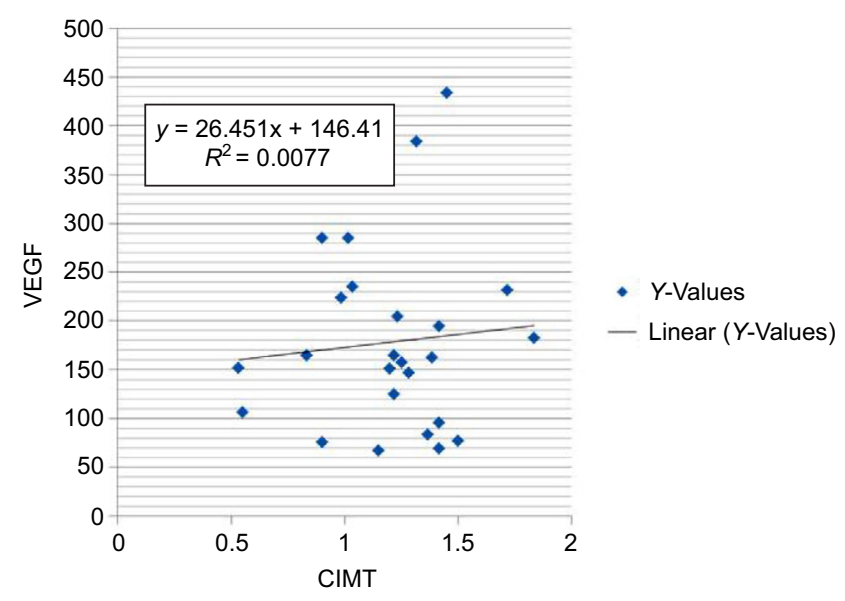

Figure I Curve of linear correlation between CIMT and plasma VEGF. Note: Demonstrates linear correlation between CIMT and plasma VEGF levels with $R$-value of $0.78 \%$.

Abbreviation: CIMT, carotid intima-media thickness.

responses in the atherosclerotic process, such as the role of PF4 which inhibits the degradation of low-density lipoprotein (LDL), facilitating the oxidation of LDL, recruiting monocytes, and facilitating their differentiation into macrophages. In addition, platelet factors also act as an anti-angiogenic chemokine by inhibiting VEGF. ${ }^{8}$ Differences in the theory of relationship between atherosclerosis and VEGF emphasize the examination of the relationship between atherosclerosis and plasma VEGF levels assessed by carotid artery ultrasound.

Angiogenesis denotes a vital process in the growth and development of tissues which also takes place in pathological conditions, such as stroke, malignancy, inflammation, and diabetes mellitus. ${ }^{7,8}$ Currently, treatment for stroke which amplifies angiogenic and neurogenic processes has been developed. ${ }^{9}$ In Indonesia, the blood test for VEGF is not yet widely available. This research aims at describing the effects of atherosclerosis examined through carotid intima-media thickness (CIMT) on the angiogenic process examined through the levels of VEGF, which seems to be able to assist clinicians in assessing vascular health, so as to provide management and selection of appropriate therapy.

\section{Methods}

\section{Research subjects}

The research design was observational analytic. The data were collected cross-sectionally from in patients and out patients with post-acute thrombotic stroke in days 7 to 90 in Dr. Saiful Anwar General Hospital Malang, Indonesia. The research subjects were 25 patients who were chosen consecutively.

\section{Inclusion criteria}

- Patients with clinically diagnosed post-acute thrombotic stroke identified through computed tomography scan or magnetic resonance imaging.

- Patients must be willing to participate in the research (by filling in informed consent form).

- For patients with loss of consciousness/Glasgow Coma Scale of less than 15 , the consent was obtained from authorized family.

\section{Exclusion criteria}

- Patients who had received intra-arterial thrombolytic therapy.

- Patients who were agitated which causes difficulties in carotid ultrasound examination.

- Patients with myocardium infarction and malignancy.

\section{Place and time of research}

This research was conducted at Radiology and Neurology Units for in patients and out patients at Dr. Saiful Anwar General Hospital Malang and at Physiology Laboratory of Faculty of Medicine, Universitas Brawijaya.

\section{Materials and tools}

Materials and tools used in this research were USG GE Logiq S6 with linear probe (5-13 MHz) and enzyme-linked immunosorbent assay (ELISA) with VEGF-A Elab science kit.

\section{Working technique and informed consent}

This research was approved by the Medical Research Ethics Committee of Dr. Saiful Anwar General Hospital Malang with certificate no. 400/12/K.3/302/2016. Patients provided written informed consent prior to the examination and participation in this study.

\section{Procedures}

Carotid ultrasonography was performed under the supervision of two radiology specialists with background experience of more than 5 years. Measurements were taken at the bilateral carotid artery in three sites, namely the common carotid artery ( $1 \mathrm{~cm}$ distal segments before the bulb), the bulb, and the proximal internal carotid artery $(1 \mathrm{~cm}$ proximal segment after branching) on the thickest CIMT by excluding plaque protruding into intravascular. The results were averaged.

In the same day, an amount of 3 cc venous blood sampling was performed on cubital vein location and then put into ethylenediaminetetraacetic acid tubes. The blood was then 
centrifuged and kept frozen until all the samples were collected and analyzed concurrently using ELISA with VEGF-A Elabscience kit at the Physiology department of University of Brawijaya.

Secondary data were obtained from medical records of patients comprising ages, the onset of stroke, results of blood lipid examination, previous stroke history, and history of diabetes mellitus.

\section{Statistical analysis}

All the data were recorded in a log book and stored as computer files. The relations between CIMT and plasma VEGF levels were tested using Pearson correlation with confidence level of $95 \%, \alpha=0.05$, and is significant when $p<0.05$.

\section{Results}

In a 3-month period, 25 research subjects who met the inclusion criteria were gained. They comprised $40 \%$ male and $60 \%$ female patients. The age ranged from 46 to 82 years old with the majority ranging from 51-60 years (40\%). For most patients $(88 \%)$ this was their first stroke and $24 \%$ of patients had a history of diabetes mellitus (Table 1).

Table I Distribution of patients based on research variables

\begin{tabular}{lll}
\hline Variable & Amount & Percentage \\
\hline CIMT (n=25) & 3 & 12 \\
Normal & 19 & 88 \\
Thickening & & \\
VEGF (n=25) & 0 & 0 \\
Normal & 25 & 100 \\
Increased & & \\
Total cholesterol $(\mathbf{n}=\mathbf{2 2})$ & 14 & 63.6 \\
Normal & 8 & 36.7 \\
Increased & & \\
Triglycerides $(\mathbf{n}=\mathbf{2 2})$ & 16 & 72.7 \\
Normal & 6 & 27.3 \\
Increased & & \\
HDL (n=21) & 3 & 14.3 \\
Normal & 18 & 85.7 \\
Decreased & & 14.3 \\
LDL (n=21) & 3 & 85.7 \\
Normal & 18 & Increased
\end{tabular}

Abbreviations: CIMT, carotid intima-media thickness; HDL, high-density lipoprotein; LDL, low-density lipoprotein.

Table 2 Correlation between CIMT and plasma VEGF levels

\begin{tabular}{ll}
\hline CIMT & Transformed VEGF \\
\hline Pearson correlation & 0.07 \\
Sig (2-tailed) & $0.74 \mathrm{I}$ \\
$\mathrm{N}$ & 25 \\
\hline
\end{tabular}

Note: Significant if $p<0.05$ (Pearson).

Abbreviation: CIMT, carotid intima-media thickness.
Following data normality test, Pearson correlation test was performed which resulted in the coefficient and significance as shown in Table 2.

The result presented in Table 2 indicates no apparent significant correlation between CIMT and plasma VEGF levels $(p=0.741)$.

Table 3 demonstrates the means of CMIT assessed with one-way analysis of variance which resulted in a significant difference by age group. Comparative analysis on multiple post hoc tests exhibits a result of significant difference in the age group $<50$ years and $51-60$ years old $(p=0.005)$, while the differences in other age groups were not significant. Table 3 also displays no difference in CIMT by sex, history of diabetes mellitus, and earlier stroke.

From Table 4, it can be seen that there is no significant difference based on the average VEGF by sex, age group, history of diabetes mellitus, and history of stroke with $p>0.05$.

Table 5 exhibits a significant correlation between CIMT with total cholesterol and LDL with $p<0.05$. As for profile of

Table 3 Comparison of average CIMT by sex, age group, history of diabetes, and history of previous stroke

\begin{tabular}{lc}
\hline Parameter & $P$-value \\
\hline Sex & $0.228^{\mathrm{a}}$ \\
Age group & $0.037^{*, \mathrm{~b}}$ \\
DM history & $0.134^{\mathrm{a}}$ \\
History of previous stroke & $0.540^{\mathrm{a}}$ \\
\hline
\end{tabular}

Notes: ${ }^{2}$ Assessed with independent samples $t$-test, ${ }^{b}$ assessed with one-way ANOVA. *Significant if $p<0.05$.

Abbreviations: CIMT, carotid intima-media thickness; DM, diabetes mellitus; ANOVA, analysis of variance.

Table 4 Comparison of average VEGF by sex, age group, history of diabetes, and history of previous stroke

\begin{tabular}{ll}
\hline Parameter & $P$-value \\
\hline Sex & $0.581^{\mathrm{a}}$ \\
Age group & $0.380^{\mathrm{b}}$ \\
DM history & $0.320^{\mathrm{a}}$ \\
History of previous stroke & $0.893^{\mathrm{a}}$ \\
\hline
\end{tabular}

Notes: ${ }^{A}$ Assessed with independent samples $t$-test, ${ }^{b}$ assessed with one-way ANOVA. Significant if $p<0.05$.

Abbreviations: DM, diabetes mellitus; ANOVA, analysis of variance.

Table 5 Correlation between CIMT and lipid profile

\begin{tabular}{lllll}
\hline CIMT & Total cholesterol & LDL & THDL & TTG \\
\hline Pearson correlation & $0.465^{*}$ & $0.510^{*}$ & 0.166 & 0.082 \\
Sig (2-tailed) & 0.029 & 0.018 & 0.472 & 0.716 \\
$\mathrm{~N}$ & 22 & 21 & 21 & 22 \\
\hline
\end{tabular}

Note: *Significant correlations with $p<0.05$.

Abbreviations: CIMT, carotid intima-media thickness; LDL, low-density lipoprotein; THDL, transformed high-density lipoprotein;TTG, transformed triglycerides. 
other fats, such as high-density lipoprotein and triglycerides, no significant correlation emerges. Figure 1 showed correlation beetwen CIMT and plasma VEGF and Figure 2 showed sonographic examination in one patient.

\section{Discussion}

In this study, from a total of 25 patients, CIMT thickening was diagnosed in 22 patients (88\%). CIMT thickening is not directly related to the incidence of stroke. Instead, it is associated with the increased risk of stroke. The measurement illustrates what happens to blood vessels per individual. Similarly, the rate of change in CIMT in a certain period of time illustrates how the development of atherosclerosis changes at all times. ${ }^{10}$

Based on the results of Pearson correlation test, there was no significant correlation between plasma VEGF levels and the means of CIMT measurements obtained from six locations: right and left common carotid arteries, right and left bulbs, and right and left internal carotid arteries. This can be explained by a number of possibilities. The increased plasma VEGF was stimulated by nonspecific processes of tissue ischemia. VEGF synthesis is stimulated when cells undergo oxygen or glucose deficiency, or in inflammatory reactions. ${ }^{11}$ In thrombotic stroke, both ischemia in blood vessels and ischemia in brain tissues occur and provoke VEGF expressions. Research by Yulinda et al examined a correlation between IMT thickness and VEGF in a mouse model in which aortic IMT was examined under microscopes, and VEGF levels were examined with immunohistochemical methods resulting in more specific description of VEGF levels in blood vessels. ${ }^{12}$ This study did not allow the examination of VEGF by immunohistochemistry. Yet, another study by
Hiyama et al used subject patients with endarterectomy in order that VEGF could be examined immunohistochemically and a correlation between the characteristics of the plaque with VEGF expression could be acquired. ${ }^{13}$

In terms of statistical analysis, all research samples showed elevated levels of plasma VEGF and most had CIMT thickening. Differences in the values among abnormal levels were not regarded as significant. If an $\mathrm{x}$, y scatter graph was made to illustrate the linearity of the relationship between CIMT with plasma VEGF levels, an $R^{2}$-value of 0.007 would be obtained accordingly. That means only $0.7 \%$ of the relationships can be explained using these variables, while the remaining $99.3 \%$ of the relationships must be explained through other variables that were not examined in the present research.

International reference for VEGF preparation has not been enforced thus far. Immunoassays are preferred in clinical practices even though it may just detect VEGF epitopes with no biological activities. The emphasis is that ELISA antibody selectively detects free VEGF. Changes in the concentration of free VEGF genuinely describe the production of relative VEGF toward degradation or the change in the binding of protein carrier. ${ }^{11}$ However, this study had employed VEGF levels in plasma, instead of serum, in which the release of VEGF in the platelets and other blood cells during the coagulation process occurred. More careful observations of the concentration of VEGF serum were able to provide further illustrations about the amount of blood platelet in the synthesis of peripheral tissues. ${ }^{11}$

The results of this research did not indicate significant differences in the means of CIMT according to history of previous stroke(s). Still, the mean values of CIMT in patients with a

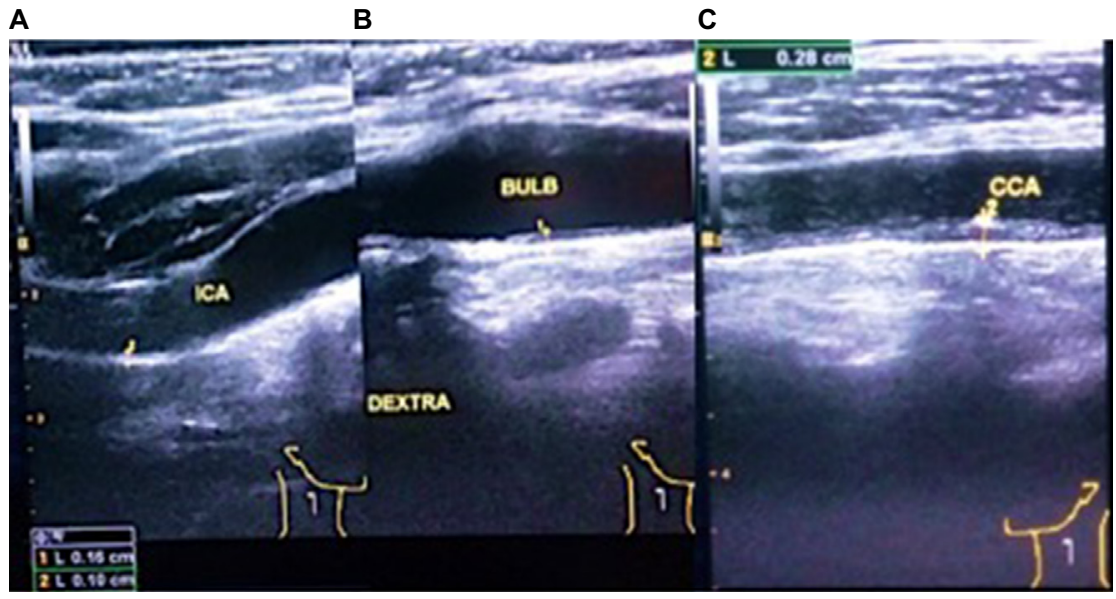

Figure 2 Sonographic examination of carotid arteries in one patient, from this figure we can see intima-media thickness measurement, carotid bulb measurement, and spectral Doppler.

Notes: (A) IMT measurements in proximal ICA; (B) measurement of the carotid bulb; (C) IMT measurements on CCA.

Abbreviations: IMT, intima-media thickness; ICA, internal carotid artery; CCA, common carotid artery. 
history of more than one stroke were descriptively discovered to be higher $(1.311 \pm 0.258 \mathrm{~mm})$ compared to the history of first stroke $(1.190 \pm 0.321 \mathrm{~mm})$. This is in conformity with the Rotterdam study which involved 7,983 healthy subjects. ${ }^{9}$ They used CIMT measurements followed by stroke incidence for an average follow-up period of 2.7 years, from which gradual increased risks of stroke incidence due to increased CIMT were detected. Odds ratio for stroke per $0.163 \mathrm{~mm}$ standard deviation increase was $1.41 .^{9}$ Similarly, the result of Eigenbrodt et al's study revealed that CIMT thickening is related to the risk of stroke even after an adjustment of stroke risk factors (hazard ratio $[\mathrm{HR}]=3.7,95 \%$ confidence interval $[\mathrm{CI}]=1.9-7.4$ in males and $\mathrm{HR}=5.7,95 \% \mathrm{CI}=1.7-19$ in females). ${ }^{10}$ Insignificance of the statistical analysis in this study may be caused by differences in the design as the Rotterdam study was a cohort study.

Similarly, differences in the mean values of CIMT were assessed based on the history of diabetes mellitus. This study found no significant difference between the mean values of CIMT in patients with a diabetes history and in patients with no diabetes history. A study by Lee et al found an association between increased CIMT and plaque score with ischemic stroke in patients with diabetes mellitus. However, after the adjustment for risk factors for cerebrovascular disease, CIMT, and plaque no longer had a significant association with ischemic stroke. ${ }^{14}$ This descriptive study found that average CIMT in patients with diabetes was higher $(1.372 \pm 3.88 \mathrm{~mm})$ than in patients with no history of diabetes $(1.151 \pm 2.75 \mathrm{~mm})$.

The role of VEGF and low-intensity inflammatory process in post-acute ischemic stroke patients remains controversial. A study by Berezin and Lisovaya suggested that increased circulating VEGF was associated with cumulative cardiovascular incidence within 1 year. ${ }^{15}$ Research by Matsuo et al argued that higher VEGF levels in atherothrombotic stroke, therefore, were associated with better outcomes than the low levels of VEGF in post-acute thrombotic stroke. Further, the research found that high levels of VEGF in embolic stroke were associated with worse outcomes. ${ }^{16}$ In this current study, evaluation of patients' clinical conditions was not performed. Likewise, the cross-sectional research design did not lead to finding information about the relationship between VEGF and clinical outcomes of patients. The plausibility in embolic stroke is that the wider area of ischemic brain and the higher of VEGF expressions which resulted in worse clinical outcome.

In this present study, a significant difference in the average VEGF according to diabetes mellitus history was unidentified. This is in contrast with the research by Mahdy et al in which VEGF serum increased significantly in patients with diabetes mellitus with micro- and macrovascular complications. ${ }^{17}$ Even so, the descriptive statistical analysis showed a trend of increased VEGF in patients with a history of diabetes mellitus $(212.28 \pm 101.93 \mathrm{pg} / \mathrm{mL})$ compared to patients without a history of diabetes $(167.54 \pm 91.53 \mathrm{pg} / \mathrm{mL})$. These significant differences may be due to the study design distinction in which Mahdy et al employed a case-control approach.

This study identifies significant correlations between the mean values of CIMT with total cholesterol and LDL levels in blood. This can be explained as due to the process of atherosclerosis, which started with the accumulation of oxidized LDL in the matrix below the layer of endothelial cells of blood vessels which stimulates the recruitment of monocytes. ${ }^{18}$ Atherosclerotic plaques were initially seen as CIMT thickening then followed by the echogenic material coating the arterial lumen. ${ }^{19}$

The result of analysis on correlation between plasma VEGF levels and LDL provides a negative yet insignificant correlation. This is consistent with the theory which suggests that VEGF expression is inhibited by LDL. The absence of significance of correlation is probably because the blood sampling for evaluation of VEGF levels and for lipid profile was taken at different times.

\section{Conclusion}

There were no significant correlations between CIMT and plasma VEGF levels in patients with post-acute thrombotic stroke. However, there was CIMT thickening in most (88\%) patients and plasma VEGF levels increased in all patients with thrombotic stroke. CIMT measurement is a promising noninvasive modality to assess the vascular condition of patients with stroke and diabetes, while plasma VEGF cannot specifically assess vascular condition as it can be triggered by ischemic conditions in tissues of the whole body.

\section{Disclosure}

This work was supported by grants from Ministry of Health, Indonesia. The authors declare that there are no conflicts of interest regarding the publication of this paper.

\section{References}

1. Xing C, Arai K, Lo EH, Hommel M. Pathophysiologic cascades in ischemic stroke. Int J Stroke. 2012;7(5):378-385.

2. Yueniwati Y, Yurina V, Indra MR. Thicker carotid intima media thickness in children with monocyte chemoattractant protein-1: A-2138T and A-2464G mutation. Neurol Res Int. 2014;2014:176535.

3. Yueniwati Y, Yurina V, Sobah N, Rahayu E. Genetic variations in the osteopontin promoters T-443C and G-156GG increase carotid intimamedia thickness. Int J Gen Med. 2016;9:117-122.

4. Yayasan stroke indonesia. sekilas tentang stroke. 2012. Availlabel from: http://www.yastroki.or.id/read.php?id=361. Accessed October 3, 2016. 
5. Caplan LR. Diagnosis and the clinical encounter. In: Caplan's Stroke: A Clinical Approach. Elsevier Inc.; 2009:64-86.

6. Wolf PA, Kannel WB. Epidemiology of stroke. In: Stroke. 5th ed. Elsevier; 2006:198-218.

7. $\mathrm{Xu} \mathrm{J}, \mathrm{Lu} \mathrm{X}, \mathrm{Shi} \mathrm{G}$. Vasa vasorum in atherosclerosis and clinical significance. Int J Mol Sci. 2015;16(5):11574-11608.

8. Aidoudi S, Bikfalvi A. Interaction of PF4 (CXCL4) with the vasculature: a role in atherosclerosis and angiogenesis. Thromb Haemost. 2010;104(5): 941-948.

9. Bots ML, Hoes AW, Koudstaal PJ, Hofman A, Grobbee DE. Common carotid intima-media thickness and risk of stroke and myocardial infarction: the Rotterdam Study. Circulation. 1997;96(5):1432-1437.

10. Eigenbrodt ML, Evans GW, Rose KM, et al. Bilateral common carotid artery ultrasound for prediction of incident strokes using intima-media thickness and external diameter: an observational study. Cardiovasc Ultrasound. 2013;11:22.

11. Jelkmann W. Pitfalls in the measurement of circulating vascular endothelial growth factor. Clin Chem. 2001;47(4):617-623.

12. Yulinda D, Yueniwati $Y$, Nurseta T. Vigna unguiculata reduces aortic intima-media thickness and increases aortic diameter and angiogenesis in ovariectomized rats. Journal of Experimental and Integrative Medicine. 2014;4(2):85-88.
13. Hiyama T, Tanaka T, Endo S, Komine K, Kudo T, Kobayashi H, Shiokawa Y. Angiogenesis in atherosclerotic plaque obtained from carotid endarterectomy: association between symptomatology and plaque morphology. Neurol Med Chir (Tokyo). 2010;50(12):1056-1061.

14. Lee EJ, Kim HJ, Bae JM, et al. Relevance of common carotid intima-media thickness and carotid plaque as risk factors for ischemic stroke in patients with type 2 diabetes mellitus. AJNR Am J Neuroradiol. 2007;28(5): 916-919.

15. Berezin AE, Lisovaya OA. Predicted value of serial measurements of circulating vascular endothelial growth factor- 1 in arterial hypertension patients after acute ischaemic stroke. Intern Med. 2014;17(1):14-20.

16. Matsuo R, Ago T, Kamouchi M, et al. Clinical significance of plasma VEGF value in ischemic stroke - research for biomarkers in ischemic stroke (REBIOS) study. BMC Neurol. 2013;13:32.

17. Mahdy RA, Nada WM, Hadhoud KM, El-Tarhony SA. The role of vascular endothelial growth factor in the progression of diabetic vascular complications. Eye (Lond). 2010;24(10):1576-1584.

18. Moore KJ, Tabas I. Review macrophages in the pathogenesis of atherosclerosis. Cell. 2011;145(3):341-355.

19. Bluth EI. 8 - Ultrasound assessment of carotid plaque. In: Pellerito J, Polak JF. Introduction to Vascular Ultrasonography. London: Elsevier Health Sciences; 2011:147-157.
International Journal of General Medicine

\section{Publish your work in this journal}

The International Journal of General Medicine is an international, peer-reviewed open-access journal that focuses on general and internal medicine, pathogenesis, epidemiology, diagnosis, monitoring and treatment protocols. The journal is characterized by the rapid reporting of reviews, original research and clinical studies across all disease areas.

\section{Dovepress}

The manuscript management system is completely online and includes a very quick and fair peer-review system, which is all easy to use. Visit http://www.dovepress.com/testimonials.php to read real quotes from published authors. 